\title{
Association of microRNA-21 expression with its targets, PDCD4 and TIMP3, in pancreatic ductal adenocarcinoma
}

\author{
Yuichi Nagao ${ }^{1,2}$, Masanori Hisaoka ${ }^{1}$, Atsuji Matsuyama ${ }^{1}$, Shuichi Kanemitsu ${ }^{2}$, \\ Tetsuo Hamada ${ }^{3}$, Tokihiko Fukuyama ${ }^{4}$, Ryuji Nakano ${ }^{5}$, Akihiko Uchiyama ${ }^{6}$, \\ Masahiko Kawamoto ${ }^{6}$, Koji Yamaguchi ${ }^{2}$ and Hiroshi Hashimoto ${ }^{1}$ \\ ${ }^{1}$ Department of Pathology and Oncology, School of Medicine, University of Occupational and Environmental \\ Health, Kitakyushu, Japan; ${ }^{2}$ Department of Surgery 1, School of Medicine, University of Occupational and \\ Environmental Health, Kitakyushu, Japan; ${ }^{3}$ Department of Surgical Pathology, Kyushu Rosai Hospital, \\ Kitakyushu, Japan; ${ }^{4}$ Department of Surgery, Kyushu Rosai Hospital, Kitakyushu, Japan; ${ }^{5}$ Department \\ of Pathology, Kyushu Kosei-Nenkin Hospital, Kitakyushu, Japan and ${ }^{6}$ Department of Surgery, Kyushu
} Kosei-Nenkin Hospital, Kitakyushu, Japan

Since the discovery of small non-coding RNAs, the analyses of microRNA (miRNA) expression patterns in human cancer have provided new insights into cancer biology. miRNA-21 has been suggested to be one of the miRNAs that have an important role in the development or biological behavior of a variety of malignancies, including pancreatic cancer. This study was conducted to evaluate the relationship between the expression of miRNA-21 and that of its molecular targets, programmed cell death 4 (PDCD4) and tissue inhibitor of metalloproteinase (TIMP3), in pancreatic ductal adenocarcinoma. The study included 65 pancreatic ductal adenocarcinomas and 5 normal pancreatic tissue specimens for comparison. The miRNA expression profiling of five selected pancreatic ductal adenocarcinomas and five normal pancreatic specimens was performed using a microarray platform, and was evaluated by a hierarchical clustering analysis. The miRNA most highly expressed in pancreatic ductal adenocarcinomas (ie, miRNA-21) was further assessed by quantitative real-time reverse transcription PCR (RT-PCR) assays in the 65 pancreatic ductal adenocarcinoma cases. The expression pattern of its molecular targets (eg, PDCD4 and TIMP3) in pancreatic ductal adenocarcinoma was examined immunohistochemically. In the microarray analyses, 28 miRNAs were upregulated in pancreatic ductal adenocarcinoma compared with normal pancreatic tissue, whereas 48 miRNAs were downregulated. miRNA-21 was the most significantly overexpressed miRNA in the pancreatic ductal adenocarcinomas analyzed, and was also highly expressed in $75 \%$ of the 65 pancreatic ductal adenocarcinomas examined by real-time RT-PCR. High miRNA-21 expression was correlated with a worse prognosis in the pancreatic ductal adenocarcinoma patients $(P=0.045)$. The immunohistochemical expression patterns of PDCD4 (reduced nuclear staining pattern) and TIMP3 (downregulated expression) were significantly associated with both the upregulated miR-21 expression $(P<0.05)$ and the poor survival of the patients $(P<0.001$ and $P=0.001$, respectively). Our data suggest that an overexpression of miRNA-21 is, therefore, associated with the biological behavior of pancreatic ductal adenocarcinoma via the downregulation of the expression of tumor suppressors, PDCD4 and TIMP3, thus resulting in tumor progression and the adverse clinical course of pancreatic ductal adenocarcinoma.

Modern Pathology (2012) 25, 112-121; doi:10.1038/modpathol.2011.142; published online 7 October 2011

Keywords: pancreatic cancer; microRNA; expression profiling; programmed cell death 4; tissue inhibitor of metalloproteinase 3

Correspondence: Dr M Hisaoka, MD, Department of Pathology and Oncology, School of Medicine, University of Occupational and Environmental Health, 1-1 Iseigaoka, Yahatanishi-ku, Kitakyushu 807-8555, Japan.

E-mail: hisaoka@med.uoeh-u.ac.jp

Received 6 April 2011; revised 20 July 2011; accepted 21 July 2011; published online 7 October 2011 
Pancreatic ductal adenocarcinoma is the fourth leading cause of cancer-related death, with only $6 \%$ of patients alive 5 years after diagnosis. ${ }^{1}$ The main reasons for this grim prognosis include the late clinical presentation, rapid and aggressive local invasion, high metastatic potential and strong resistance to chemotherapy and radiation therapy of pancreatic ductal adenocarcinomas. Although the only potentially curative treatment for pancreatic ductal adenocarcinoma is surgical resection, the vast majority of patients are diagnosed at too advanced stages to undergo a curative surgery. This late diagnosis stems largely from a paucity of disease-specific symptoms in the early stages. Although some previous studies have attempted to identify predictive markers of pancreatic ductal adenocarcinoma, no single predictive marker is so far available. ${ }^{2}$ In addition, the molecular mechanisms underlying such biological aggressiveness of pancreatic ductal adenocarcinoma have not yet been fully elucidated.

A recently identified class of non-coding small RNAs, microRNAs (miRNAs), is a group of RNAs of 14-24 nucleotides that can negatively regulate protein expression at the post-transcriptional level by translational inhibition and/or mRNA degradation, mostly through base pairing with the $3^{\prime}$-UTR of their target mRNAs. ${ }^{3}$ Accumulated bioinformatic data indicate that a single miRNA can potentially bind to hundreds of mRNA targets, thereby having an important role in various biological processes. ${ }^{4}$ Furthermore, it has been demonstrated that miRNAs can induce tumorigenesis, for example, by downregulating tumor suppressor genes. Among such oncogenic miRNAs, miRNA-21 (miR-21) has been shown to be overexpressed in a variety of malignancies, including pancreatic cancer, ${ }^{5,6}$ esophageal cancer, ${ }^{7}$ lung cancer, ${ }^{8}$ and colon cancer. ${ }^{9}$ miR-21 has been shown to target tumor suppressor genes such as those of tropomyosin 1 (TPM1), programmed cell death 4 (PDCD4), phosphatase and tensin homolog (PTEN), tissue inhibitor of metalloproteinase 3 (TIMP3), and Maspin. ${ }^{10-12}$ However, the relationship between miR-21 and such tumor suppressor genes remains to be investigated in pancreatic ductal adenocarcinoma.

The aims of this study were to evaluate the miRNA expression patterns and to assess the potential relationship between the expression levels of miR-21 and its putative molecular targets, TIMP3 and PDCD4, and the clinicopathological significance of miR-21 expression in pancreatic ductal adenocarcinoma.

\section{Materials and methods}

\section{Patients and Tissue Samples}

The study included 65 patients with pancreatic ductal adenocarcinoma who underwent surgical resection in the Department of Surgery 1, School of Medicine, University of Occupational and Environ- mental Health, Japan; the Department of Surgery, Kyusyu Rosai Hospital; and the Department of Surgery, Kyushu Kosei-Nenkin Hospital in the period from 1982 to 2010. The patients comprised 40 males and 25 females, with a median age of 65 years (range, 40-80 years). Survival was measured from the time of the pancreatic resection, and death was regarded as an end point. The survival information of the patients followed-up at least 0.5 years after surgery was obtained. Formalin-fixed, paraffin-embedded tumor tissue specimens were re-cut and stained with hematoxylin and eosin for a histological re-evaluation according to the current criteria of the World Health Organization. ${ }^{13}$ The tumor stage was assessed based on the Union Internationale Contre le Cancer (UICC) classification. ${ }^{14}$ This study included cases of 'pT1-3 No-1 M0' and 'pT4 No Mo' disease. The detailed clinical data of these cases are shown in Table 1. Written informed consent was obtained from all patients before sample collection, and all patients were treatment naive before pancreatic surgery.

Table 1 Clinicopathological profiles of 65 pancreatic ductal adenocarcinoma patients

\begin{tabular}{lc}
\hline Sex (male/female) & $40(62 \%) / 25(38 \%)$ \\
Median age & 65 years (range, $40-80$ years) \\
pT category & \\
pT1 & $3(5 \%)$ \\
pT2 & $9(14 \%)$ \\
pT3 & $46(71 \%)$ \\
pT4 & $7(10 \%)$ \\
pN category & \\
pNo & \\
pN1 & $36(55 \%)$ \\
pM category & $29(45 \%)$ \\
pM0 & \\
pM1 & $65(100 \%)$ \\
Histology & $0(0 \%)$ \\
Well differentiated & \\
Moderate differentiated & \\
Poorly differentiated & $16(24 \%)$ \\
& $42(65 \%)$ \\
Location & $7(11 \%)$ \\
Head & \\
Body & \\
Lymphatic permeation & \\
None or slight & \\
Prominent & $18(72 \%)$ \\
Vascular invasion & \\
None or slight & \\
Prominent & \\
Perineural invasion & \\
None or slight & \\
Prominent & $37(52(50 \%)$ \\
Tumor diameter & \\
$\leq 4$ cm & \\
$>4$ cm & \\
\hline
\end{tabular}


Snap-frozen tumor tissues of five randomly selected cases were used in an miRNA microarray study. Formalin-fixed, paraffin-embedded tumor tissues of the other 60 cases were used for reverse transcription PCR (RT-PCR) and an immunohistochemical study. Normal pancreatic tissue specimens from five autopsy cases were included for comparison purposes.

\section{RNA Extraction and miRNA Microarrays}

Small RNAs extracted from the tissue specimens were purified and labeled with Cy5 using the Label IT miRNA labeling kit (Takara Bio, Otsu, Japan). Expression profiles of miRNA were analyzed using a 3D-Gene miRNA microarray platform (version 14.0, Toray, Kamakura, Japan) as described previously. ${ }^{15}$ More than 900 antisense probes were printed in duplicate spots on the miRNA microchip The annotation and oligonucleotide sequences of the probes conformed to the miRBase miRNA database version 14.0 (http://microrna.sanger.ac.uk/ sequences/). The data were assessed using unsupervised hierarchical clustering.

\section{Quantitative Estimation of miRNA by RT-PCR}

A quantitative estimation of miRNA expression was performed by real-time RT-PCR using the TaqMan MicroRNA Assay and TaqMan MicroRNA RT kit (Applied Biosystems, Foster City, CA) according to the manufacturer's instructions. Briefly, the reaction master mix containing $10 \times$ RT buffer, $5 \times$ RT primers, MultiScribe reverse transcriptase, RNase inhibitor, $100 \mathrm{mM}$ dNTPs and nuclease-free water was mixed with $20 \mathrm{ng}$ of total RNA extracted from the PDAC and normal pancreatic tissue samples. The mixtures were incubated for $30 \mathrm{~min}$ at $16^{\circ} \mathrm{C}$, $30 \mathrm{~min}$ at $42^{\circ} \mathrm{C}$, and $5 \mathrm{~min}$ at $85^{\circ} \mathrm{C}$. The PCR was performed using $10 \mu \mathrm{l}$ of PCR master mix containing TaqMan $2 \times$ Universal PCR Master Mix, $20 \times$ TaqMan MicroRNA Assay Mix (Applied Biosystems) and the RT products in a volume of $20 \mu \mathrm{l}$. The reaction mixtures were incubated at $95^{\circ} \mathrm{C}$ for $10 \mathrm{~min}$, followed by 40 cycles at $95^{\circ} \mathrm{C}$ for $15 \mathrm{~s}$ and at $60^{\circ} \mathrm{C}$ for $1 \mathrm{~min}$ using the StepOnePlus Real-Time PCR system (Applied Biosystems). The mean Ct values of each sample were determined from duplicate reactions. The relative expression level of each miRNA examined was calculated by $\log _{2}\left|2^{-\Delta C t}\right|$, in which $\Delta C \mathrm{t}$ was defined as the subtraction of the $C$ t value of the target miRNA from the $C t$ value of the internal control RNU6B.

\section{Immunohistochemical Analysis}

Unstained sections of $4 \mu \mathrm{m}$ thickness were deparaffinized in xylene and dehydrated in a graded series of alcohol, and stained according to the routine laboratory protocols. After standard antigen retrieval in a pressure cooker for $10 \mathrm{~min}$, endogenous peroxidase was blocked in 3\% hydrogen peroxide in methanol for $5 \mathrm{~min}$, followed by a treatment with a Protein Blocking Agent (DAKO Japan, Tokyo, Japan). Each incubation step was carried out at room temperature and was followed by three sequential washes in TBS. Sections were incubated with a primary antibody, anti-PDCD4 (1:100, ab51495, Abcam, Cambridge, MA) or antiTIMP3 (1:100, ab58804, Abcam) overnight, followed by incubation with an anti-mouse secondary antibody-conjugated immunoenzyme polymer reagent (EnVision, DAKO Japan) for $30 \mathrm{~min}$. The reaction products were visualized using 3, $3^{\prime}$-diaminobenzidine tetrahydrochloride and $\mathrm{H}_{2} \mathrm{O}_{2}$. Normal pancreatic tissue samples were used as an internal positive control. The primary antibody was omitted in the staining procedure for negative controls.

The immunostaining was scored according to the percentage of positive cells by two observers, and were classified as: $(-)$ negative staining, $<30 \% ;(+)$ moderate positive staining, $30-70 \%$; and $(++)$ strong positive staining, $>70 \%$. TIMP3 expression was evaluated by its cytoplasmic staining, whereas PDCD4 expression was assessed by its both cytoplasmic and nuclear staining.

\section{Statistical Analyses}

The data about miRNA expression determined by RT-PCR were assessed using Welch-based $t$-tests. The association between the postoperative survival and the miRNA expression level measured by RTPCR was estimated by the Kaplan-Meier method, and the resulting curves were split into two classes (high and low expression in comparison with the mean level of miRNA expression as the threshold) and compared using log-rank test. Significance was accepted with 95\% confidence. Postoperative survival was calculated as the interval between the date of pancreatic cancer surgery and either the date of the patient's death or the date of the last follow-up of the patient. The $\chi^{2}$-test was used to analyze the relationship between the miR-21 expression or immunohistochemical expression of PDGD4 or TIMP3 and various clinicopathological parameters. The univariate or multivariate Cox hazard regression model analysis was also performed to assess miRNA expression and immunohistochemical data and variable clinicopathological parameters as potential predictors. The statistical analyses were performed using the SPSS 17.0 for Windows software package (SPSS Inc.). Differences were considered to be statistically significant when the $P$-value was $<0.05$.

\section{Results}

\section{miRNA Microarray Analysis}

In our global miRNA expression analysis, supervised hierarchical clustering showed distinctive 
miRNA expression profiles for each sample type, and all but one sample was properly clustered into the appropriate sample category (Figure 1). In a comparison of miRNA expression between five pancreatic ductal adenocarcinomas and five normal pancreatic tissues, 76 miRNAs were differentially expressed by at least 2-fold: 28 miRNAs were upregulated and 48 miRNAs were downregulated in pancreatic ductal adenocarcinomas compared with normal tissue samples. The 10 most overexpressed or underexpressed miRNAs are listed in
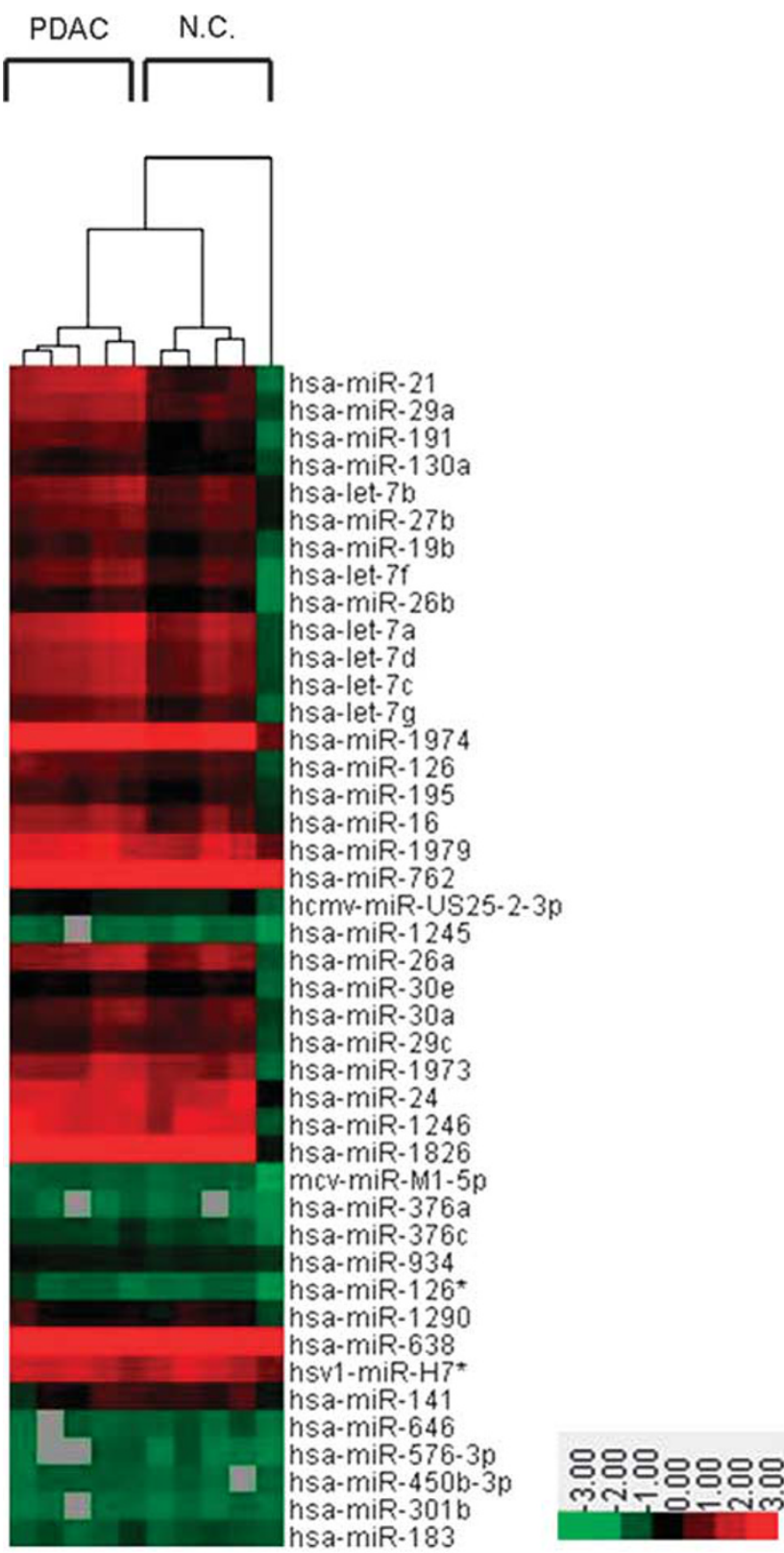

Figure 1 A partial heat map of the global miRNA expression profiles and unsupervised hierarchical clustering of five pancreatic ductal adenocarcinomas (PDAC) and five normal pancreatic tissues (N.C.). The red and green colors represent a relatively high and low expression of miRNAs, respectively.
Table 2. Among the upregulated miRNAs in pancreatic ductal adenocarcinomas, miR-21 was shown to be the most highly overexpressed miRNA, and its averaged expression level was 3.63-fold greater in pancreatic ductal adenocarcinoma than in normal pancreatic tissue samples. Therefore, the current study focused on miR-21.

\section{Quantitative Estimation of miRNA Expression by RT-PCR}

Consistent with the microarray data, most of the pancreatic ductal adenocarcinoma specimens (75\%) showed increased levels of miR-21 (mean: 1.99-fold) compared with normal pancreatic tissue $(P=0.024$; Figure 2). A statistically significant correlation between an elevated miR-21 level and lymph node metastasis was found, although other clinicopathological parameters including gender, age, $\mathrm{T}$ stage, histological grade, location, lymphatic permeation, vascular invasion, perineural invasion, and tumor size were not correlated with the miR-21 expression (Table 3).

\section{PDCD4 and TIMP3 Immunostaining}

The non-neoplastic ductal epithelium showed unequivocal or intense nuclear PDCD4 and cytoplasmic TIMP3 expression. Atypical ductal epithelium or carcinoma cells in pancreatic ductal adenocarcinoma displayed variable but often reduced nuclear and/or predominantly cytoplasmic PDCD4 staining, whereas TIMP3 staining was mostly weak or lost in many pancreatic ductal adenocarcinomas (Figure 3). However, there was no significant correlation between the immunohistochemical expression of PDCD4 or TIMP3 and the clinicopathological parameters of the pancreatic ductal adenocarcinoma cases examined (Table 4).

\section{Correlation between miR-21 and PDCD4/TIMP3 Expression}

A statistically significant correlation was found between the miR-21 expression level and downregulated nuclear PDCD4 expression $(P=0.023)$ or cytoplasmic TIMP3 expression $(P=0.027)$ in pancreatic ductal adenocarcinoma samples (Table 5). In addition, the carcinoma cells tended to express more cytoplasmic PDCD4, although its correlation with miR-21 expression was not significant.

\section{Statistical Analysis of Patient Survival}

In Kaplan-Meier survival analysis, there was a trend for patients with high miR-21 expression to have a poorer course of survival compared with those with low miR-21 expression ( $P=0.045$; Figure 4$)$. A unvariate Cox hazard regression model analysis 
Table 2 Differentially expressed miRNAs identified in pancreatic ductal adenocarcinoma

\begin{tabular}{|c|c|c|c|c|c|}
\hline \multicolumn{3}{|c|}{ miRNAs upregulated } & \multicolumn{3}{|c|}{ miRNAs downregulated } \\
\hline Gene ID & Fold change & $\mathrm{P}$-value & Gene ID & Fold change & $\mathrm{P}$-value \\
\hline has-miR-21 & 3.63 & $<0.001$ & has-miR-216a & -4.84 & 0.001 \\
\hline has-miR-199a-3p & 3.38 & $<0.001$ & has-miR-216b & -4.73 & 0.001 \\
\hline has-miR-194 & 3.29 & $<0.001$ & has-miR-217 & -4.65 & 0.003 \\
\hline has-miR-10a & 3.15 & $<0.001$ & has-miR-184 & -4.27 & $<0.001$ \\
\hline has-miR-143 & 3.14 & $<0.001$ & has-miR-423-5p & -3.71 & $<0.001$ \\
\hline has-miR-100 & 2.95 & $<0.001$ & has-miR-25 & -3.67 & $<0.001$ \\
\hline has-miR-99a & 2.9 & 0.001 & has-miR-491-5p & -3.6 & $<0.001$ \\
\hline has-miR-222 & 2.82 & $<0.001$ & has-miR-H2-3p & -3.25 & $<0.001$ \\
\hline has-miR-31 & 2.74 & 0.010 & has-miR-1292 & -3.2 & $<0.001$ \\
\hline has-miR-155 & 2.57 & $<0.001$ & has-miR-2110 & -3.19 & $<0.001$ \\
\hline
\end{tabular}

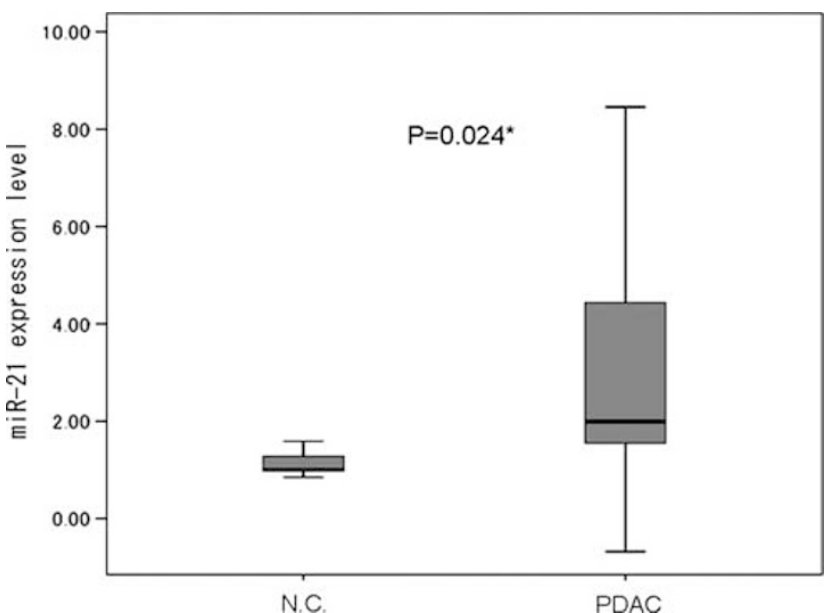

Figure 2 The miR-21 expression in normal pancreatic tissue (N.C.) and pancreatic ductal adenocarcinoma (PDAC) assessed by real-time RT-PCR. Box plots indicate the medians with interquartile range, and whiskers demonstrate the maximum and minimum levels of miR-21 expression. Statistical significance was calculated using the Welch-based $t$-test.

demonstrated that a high expression level of miR-21 $(P=0.045$, hazard ratio $(\mathrm{HR})=0.431 ; 95 \%$ $\mathrm{CI}=0.221-0.839), \mathrm{T}$ stage $(P=0.023, \mathrm{HR}=3.924$; $95 \% \mathrm{CI}=1.207-12.755)$, PDCD4 nuclear staining $(P=0.00, \mathrm{HR}=0.440 ; 95 \% \mathrm{CI}=0.282-0.689)$, and TIMP3 staining $(P=0.001, \mathrm{HR}=0.528 ; 95 \% \mathrm{CI}=$ 0.363-0.769) was associated with poor patient survival. In a multivariate Cox regression model analysis, a high expression level of miR-21 $(P=0.030$, $\mathrm{HR}=2.124 ; 95 \% \mathrm{CI}=1.074-4.200)$ and downregulated TIMP3 expression $(P=0.000, \quad \mathrm{HR}=4.732$; $95 \%=$ CI 2.245-9.974) were considered significantly unfavorable prognostic factors (Table 6).

\section{Discussion}

Dysregulated expression of miRNAs has been identified in a variety of human malignancies and is suggested to have a significant role in the development or progression of tumors by inhibiting
Table 3 Comparison of clinicopathological parameters and expression level of miRNA-21 in pancreatic ductal adenocarcinoma

\begin{tabular}{|c|c|c|c|c|}
\hline \multirow[t]{2}{*}{ Parameter } & \multirow[t]{2}{*}{$\mathrm{N}$} & \multicolumn{2}{|c|}{$\operatorname{miR}-21$} & \multirow[t]{2}{*}{$\mathrm{P}$-value } \\
\hline & & $\begin{array}{c}\text { High } \\
\text { expression }\end{array}$ & $\begin{array}{c}\text { Low } \\
\text { expression }\end{array}$ & \\
\hline \multicolumn{5}{|l|}{ Sex } \\
\hline Male & 40 & 19 & 21 & 0.724 \\
\hline Female & 25 & 13 & 12 & \\
\hline \multicolumn{5}{|l|}{ Age (years) } \\
\hline$\leq 65$ & 30 & 15 & 15 & 0.909 \\
\hline$>65$ & 35 & 17 & 18 & \\
\hline \multicolumn{5}{|l|}{ pT category } \\
\hline $1 / 2$ & 12 & 4 & 8 & 0.223 \\
\hline $3 / 4$ & 53 & 28 & 25 & \\
\hline \multicolumn{5}{|l|}{$p N$ category } \\
\hline 1 & 29 & 19 & 10 & 0.018 \\
\hline 0 & 36 & 13 & 23 & \\
\hline \multicolumn{5}{|l|}{ Histology } \\
\hline Well & 16 & 8 & 8 & 0.938 \\
\hline \multicolumn{5}{|l|}{ differentiated } \\
\hline $\begin{array}{l}\text { Moderately } \\
\text { differentiated }\end{array}$ & 42 & 21 & 21 & \\
\hline $\begin{array}{l}\text { Poorly } \\
\text { differentiated }\end{array}$ & 7 & 3 & 4 & \\
\hline \multicolumn{5}{|l|}{ Location } \\
\hline Head & 47 & 23 & 24 & 0.939 \\
\hline Body & 18 & 9 & 9 & \\
\hline \multicolumn{5}{|c|}{ Lymphatic permeation } \\
\hline None or slight & 37 & 16 & 21 & 0.267 \\
\hline Prominent & 28 & 16 & 12 & \\
\hline \multicolumn{5}{|l|}{ > Vascular invasion } \\
\hline None or slight & 54 & 26 & 28 & 0.699 \\
\hline Prominent & 11 & 6 & 5 & \\
\hline \multicolumn{5}{|c|}{ Perineural invasion } \\
\hline Non or slight & 33 & 15 & 18 & 0.536 \\
\hline Prominent & 32 & 17 & 15 & \\
\hline \multicolumn{5}{|l|}{ Tumor diameter } \\
\hline$\leq 4 \mathrm{~cm}$ & 52 & 24 & 28 & 0.321 \\
\hline$>4 \mathrm{~cm}$ & 13 & 8 & 5 & \\
\hline
\end{tabular}

${ }^{\mathrm{a}} \chi^{2}$-test. 

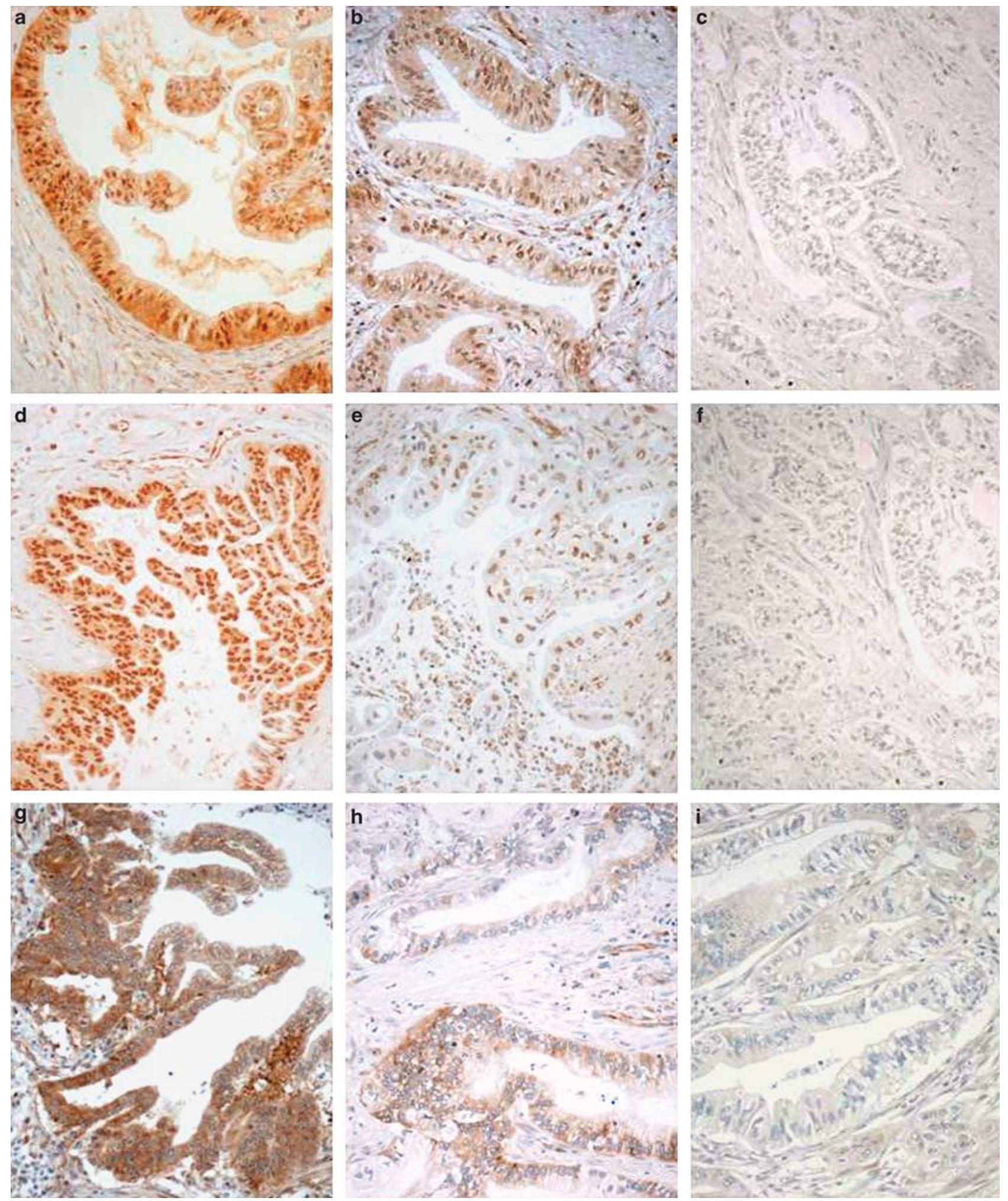

Figure 3 Immunohistochemical expression of PDCD4 (a-f) and TIMP3 (g-i) in PDAC. PDCD4 is expressed intensely (a, d) or moderately $(\mathbf{b}, \mathbf{c})$ in the cytoplasm $(\mathbf{a}, \mathbf{b})$ or nuclei $(\mathbf{d}, \mathbf{f})$ of adenocarcinoma cells, whereas it was lacking in some PDAC cases (c, f). Representative strong (g) moderate (h) or negative (i) cytoplasmic immunoreactivity for TIMP3 is also shown.

tumor suppressor genes or activating oncogenes. In pancreatic ductal adenocarcinoma or its precursor lesion, the overexpression of several miRNAs, such as miR-21, miR-181 family, miR-221 and miR-155, and downregulation of miRNAs, such as the let-7 family, miR-200 family and miR-146a, have been 
Table 4 Comparison of clinicopathological features and PDCD4 or TIMP3 expression level in pancreatic ductal adenocarcinoma

\begin{tabular}{|c|c|c|c|c|c|c|c|c|c|c|c|c|c|}
\hline \multirow[t]{2}{*}{ Clinicopathological feature } & \multirow[t]{2}{*}{$N$} & \multicolumn{4}{|c|}{ PDCD4 (cytoplasmic) } & \multicolumn{4}{|c|}{ PDCD4 (nuclear) } & \multicolumn{4}{|c|}{ TIMP3 } \\
\hline & & - & + & ++ & $\mathrm{P}$-value $\mathrm{a}^{\mathrm{a}}$ & - & + & ++ & $\mathrm{P}-v_{a l u e}{ }^{\mathrm{a}}$ & - & + & ++ & $\mathrm{P}$-value \\
\hline \multicolumn{14}{|l|}{ Sex } \\
\hline Male & 40 & 8 & 17 & 15 & 0.57 & 6 & 14 & 20 & 0.582 & 5 & 13 & 22 & 0.887 \\
\hline Female & 25 & 6 & 10 & 9 & & 3 & 12 & 10 & & 4 & 7 & 14 & \\
\hline \multicolumn{14}{|l|}{ Age (years) } \\
\hline$\leq 65$ & 30 & 5 & 14 & 11 & 0.856 & 4 & 10 & 16 & 0.535 & 5 & 9 & 16 & 0.83 \\
\hline$>65$ & 35 & 7 & 14 & 14 & & 5 & 16 & 14 & & 4 & 11 & 20 & \\
\hline \multicolumn{14}{|l|}{ pT category } \\
\hline $1 / 2$ & 12 & 1 & 5 & 6 & 0.392 & 0 & 6 & 6 & 0.293 & 0 & 2 & 10 & 0.079 \\
\hline $3 / 4$ & 53 & 13 & 22 & 18 & & 9 & 20 & 24 & & 9 & 18 & 26 & \\
\hline \multicolumn{14}{|l|}{$p N$ category } \\
\hline 0 & 36 & 8 & 12 & 9 & 0.501 & 2 & 17 & 17 & 0.079 & 5 & 10 & 14 & 0.563 \\
\hline 1 & 29 & 6 & 15 & 15 & & 7 & 9 & 13 & & 4 & 10 & 22 & \\
\hline \multicolumn{14}{|l|}{ Histology } \\
\hline Well differentiated & 16 & 3 & 8 & 5 & 0.072 & 1 & 6 & 9 & 0.721 & 3 & 3 & 10 & 0.275 \\
\hline Moderately differentiated & 42 & 11 & 18 & 13 & & 7 & 18 & 17 & & 6 & 16 & 20 & \\
\hline Poorly differentiated & 7 & 0 & 1 & 6 & & 1 & 2 & 4 & & 0 & 1 & 6 & \\
\hline \multicolumn{14}{|l|}{ Location } \\
\hline Head & 47 & 12 & 18 & 17 & 0.425 & 8 & 17 & 22 & 0.392 & 7 & 16 & 24 & 0.523 \\
\hline Body & 18 & 2 & 9 & 7 & & 1 & 9 & 8 & & 2 & 4 & 12 & \\
\hline \multicolumn{14}{|l|}{ Lymphatic permeation } \\
\hline None or slight & 37 & 8 & 16 & 13 & 0.935 & 4 & 15 & 18 & 0.707 & 6 & 11 & 20 & 0.816 \\
\hline Prominent & 28 & 6 & 11 & 11 & & 5 & 11 & 12 & & 3 & 9 & 16 & \\
\hline \multicolumn{14}{|l|}{ Venus invasion } \\
\hline None or slight & 54 & 13 & 21 & 20 & 0.474 & 6 & 22 & 26 & 0.360 & 8 & 16 & 30 & 0.838 \\
\hline Prominent & 11 & 1 & 6 & 4 & & 3 & 4 & 4 & & 1 & 4 & 6 & \\
\hline \multicolumn{14}{|l|}{ Perineural invasion } \\
\hline None or slight & 33 & 8 & 13 & 12 & 0.858 & 5 & 12 & 16 & 0.826 & 5 & 6 & 22 & 0.079 \\
\hline Prominent & 32 & 6 & 14 & 12 & & 4 & 14 & 14 & & 4 & 14 & 14 & \\
\hline \multicolumn{14}{|l|}{ Tumor diameter } \\
\hline$\leq 4 \mathrm{~cm}$ & 52 & 11 & 22 & 19 & 0.968 & 5 & 20 & 27 & 0.068 & 5 & 16 & 31 & 0.122 \\
\hline$>4 \mathrm{~cm}$ & 13 & 3 & 5 & 5 & & 4 & 6 & 3 & & 4 & 4 & 5 & \\
\hline
\end{tabular}

${ }^{\mathrm{a}} \chi^{2}$-test.

described in previous studies., ${ }^{5,12,13}$ Among them, miR-21 is the one that has been recurrently suggested to be oncogenic in the pancreas. Our microarray data about global miRNA expression further support this view, because miR-21 was the most significantly overexpressed miRNA in pancreatic ductal adenocarcinoma in our study.

Bloomston et $a l^{5}$ have described that miR-21 and miR-155 were specifically overexpressed in pancreatic cancers compared with normal pancreas or chronic pancreatitis. Since miR-21 can inhibit apoptosis, ${ }^{16}$ they assumed that miR-21 might function as a proto-oncogene. In line with this assumption, pancreatic cancer cells transfected with the miR-21 precursor show significantly increased cell proliferation, invasiveness, and chemoresistance. ${ }^{17}$ It is notable that a high miR-21 expression was associated with a poorer prognosis of the pancreatic ductal adenocarcinoma patients in the
Table 5 Correlation between miR-21 expression levels and PDCD4 or TIMP3 expression level

\begin{tabular}{cccc}
\hline & $\begin{array}{c}\text { High miR-21 } \\
\text { expression }\end{array}$ & $\begin{array}{c}\text { Low miR-21 } \\
\text { expression }\end{array}$ & P-value \\
\hline PDCD4 & (cytoplasmic) & & \\
- & $4(6 \%)$ & $10(15 \%)$ & 0.129 \\
+ & $13(20 \%)$ & $14(22 \%)$ & \\
++ & $15(23 \%)$ & $9(14 \%)$ & \\
PDCD4 & (nuclear) & & \\
- & $8(12 \%)$ & $1(2 \%)$ & \\
+ & $13(20 \%)$ & $13(20 \%)$ & \\
++ & $11(17 \%)$ & $19(29 \%)$ & \\
TIMP3 & & & \\
- & $8(12 \%)$ & $1(2 \%)$ & \\
+ & $10(15 \%)$ & $10(15 \%)$ & \\
++ & $14(22 \%)$ & $22(34 \%)$ & \\
\hline
\end{tabular}

${ }^{\mathrm{a}} \chi^{2}$-test. 
current study, and the association has also been described by Dillhoff et al. ${ }^{18}$ Moreover, the expression status of miR-21 was considered to be an independent prognostic factor for poor survival based on our statistical analysis. Zhu et $a l^{19}$ described that miR-21 has a role not only in tumor growth, but also in invasion and tumor metastasis using several cancer cell lines. We also found a significant correlation between the overexpression of miR-21 and lymph node metastasis of pancreatic ductal adenocarcinoma. These findings suggest that miR-21 can be used as a biomarker for predicting disease progression and/or the aggressiveness of pancreatic ductal adenocarcinoma. Interestingly, Ali et $a l^{12}$ recently examined miR-21 in the plasma

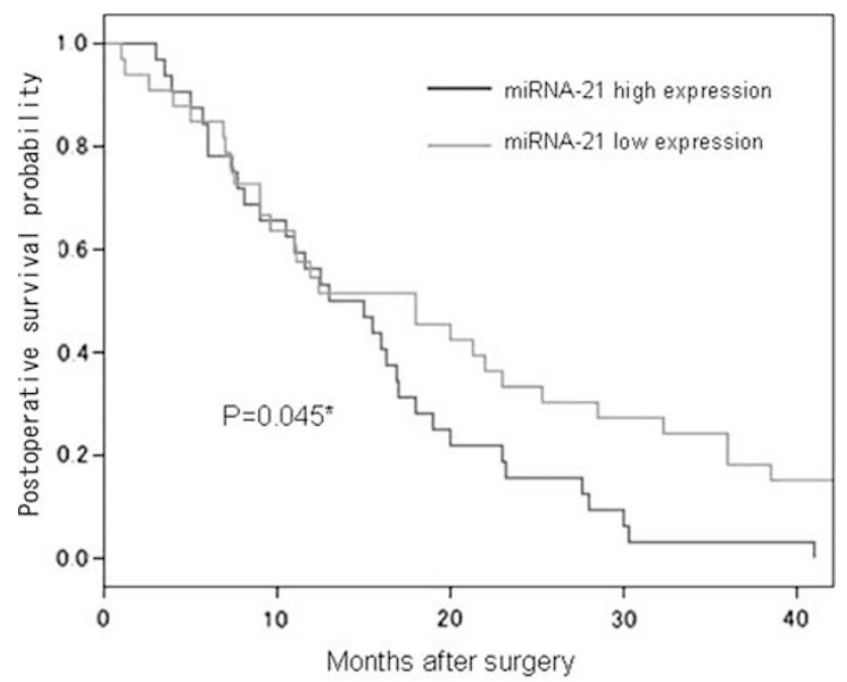

Figure 4 Kaplan-Meier overall survival analysis of patients with pancreatic ductal adenocarcinoma based on their expression of miR-21 as measured by quantitative RT-PCR. of patients with pancreatic cancer, and found that the expression miR-21 was correlated with a worse survival, which would suggest the potential practical use of plasma miR-21 in future clinical management of patients with pancreatic ductal adenocarcinoma.

Although the detailed molecular mechanisms underlying such oncogenic functions of the overexpressed miR-21 in the pancreas remain to be clarified, previous studies have identified several tumor suppressors as molecular targets of miR-21, including PTEN, PDCD4, TPM1, TIMP3, and Maspin. ${ }^{10-12}$ PTEN has been recently shown to act in synergy with Smad4 in suppressing pancreatic ductal adenocarcinoma formation in mice. ${ }^{20}$ More recently, it has been reported that inhibition of miR21 in a pancreatic ductal adenocarcinoma cell line led to reduced cell proliferation and increased cell death, with a simultaneous increase in PDCD4. ${ }^{21}$ In addition, the level of expression of PTEN, PDCD4, Maspin, and TPM1 was significantly reduced in drug-resistant pancreatic ductal adenocarcinoma cell lines. ${ }^{12}$ Our data, showing a correlation between the expression of miR-21 and its targets in pancreatic ductal adenocarcinoma tissues, are in agreement with these observations, although the association of miR-21 with TIMP3 expression has not been previously examined in the pancreas. Thus, aberrantly expressed miR-21 appears to have a pivotal role in the development and/or progression of pancreatic ductal adenocarcinoma by targeting multiple tumor suppressors.

Notably, the miR-21 expression pattern was correlated with reduced nuclear expression of PDCD4 and reduced cytoplasmic expression of TIMP3 in our study. Loss of PDCD4 has been shown to be strongly associated with the progression and metastasis of multiple human cancer types, including

Table 6 Univariate and multivariate survival analyses of the clinicopathological and molecular features of pancreatic ductal adenocarcinoma

\begin{tabular}{|c|c|c|c|c|c|}
\hline \multirow[t]{2}{*}{ Factor } & & \multicolumn{2}{|c|}{ Univariate analysis } & \multicolumn{2}{|c|}{ Multivariate analysis } \\
\hline & & $H R(95 \% C I)$ & P-value & $H R(95 \% C I)$ & $\mathrm{P}$-value \\
\hline Sex & Male vs female & $1.134(0.593-2.171)$ & 0.703 & & \\
\hline Age & $\leq 65$ vs $>65$ years & $0.741(0.383-1.331)$ & 0.289 & & \\
\hline T category & $\mathrm{T} 1 / 2$ vs $\mathrm{T} 3 / 4$ & $3.924(1.207-12.755)$ & 0.023 & & \\
\hline Lymph node metastasis & Negative vs positive & $1.709(0.923-3.165)$ & 0.088 & & \\
\hline Histology & Well vs mod vs por & $1.093(0.671-1.781)$ & 0.721 & & \\
\hline Location & Head vs Body & $0.631(0.290-1.371)$ & 0.245 & & \\
\hline Lymphatic permeation & None or slight vs prominent & $1.474(0.797-2.728)$ & 0.217 & & \\
\hline Vascular invasion & None or slight vs prominent & $2.234(0.957-5.216)$ & 0.063 & & \\
\hline Perineural invasion & None or slight vs prominent & $1.086(0.586-2.014)$ & 0.793 & & \\
\hline Tumor size & $\leq 2 \mathrm{~cm} v s>2 \mathrm{~cm}$ & $1.789(0.871-3.673)$ & 0.113 & & \\
\hline miR-21 expression & High vs low & $0.398(0.205-0.772)$ & 0.045 & $2.124(1.074-4.200)$ & 0.030 \\
\hline PDCD4 (cytoplasmic)a & $-V S+V S++$ & $0.743(0.494-1.117)$ & 0.151 & & \\
\hline PDCD4 (nuclear) ${ }^{\mathrm{a}}$ & $-v s+v s++$ & $0.440(0.282-0.689)$ & $<0.001$ & & \\
\hline TIMP3 $^{\mathrm{a}}$ & $-v s+v s++$ & $0.528(0.363-0.769)$ & 0.001 & $4.732(2.245-9.974)$ & $<0.001$ \\
\hline
\end{tabular}

${ }^{\mathrm{a}}$ Immunohistochemical expression. 
those in the lung, breast, colon, esophagus, stomach, and ovary. ${ }^{22-27}$ PDCD4 is expressed ubiquitously in normal tissues and mediates multiple biologic processes, such as cell-cycle progression and cell survival, by binding RNA and ATP-dependent RNA helicase eIF4A. ${ }^{28}$ PDCD4 shuttles between the nucleus and cytoplasm, ${ }^{29}$ and the biological significance of its subcellular localization is a matter of controversy. ${ }^{30,31}$ In our immunohistochemical study, PDCD4 was expressed exclusively in the nuclei in normal pancreatic tissue, whereas adenocarcinomas frequently showed cytoplasmic expression of PDCD4, rather than its nuclear expression. A similar trend in PDCD4 expression has been described in breast and colorectal cancers, ${ }^{10,24}$ although the pattern of PDCD4 expression somewhat differed in intraductal papillary mucinous neoplasm of the pancreas in a recent study. ${ }^{32}$ Therefore, the function and expression pattern of PDGD4 may vary according to tumor type, and the subcellular localization of PDCD4 in tumor cells warrants further investigation.

The family of TIMPs consists of four members that specifically inhibit the proteolytic activity of matrix metalloproteinases. ${ }^{33}$ TIMP3 is secreted as a $24-\mathrm{kDa}$ protein that, in contrast to other TIMP family members, binds to the extracellular matrix. TIMP3 is also considered a tumor suppressor, because this protein can inhibit tumor growth, angiogenesis, and invasion, and can promote apoptosis. ${ }^{34}$ The adenoviral transfer of TIMP3 into HeLa, HT1080 fibrosarcoma cells, and melanoma cells reduces their invasiveness and stimulates apoptosis. ${ }^{35,36}$ Strong reduction or loss of TIMP3 expression has been described in pancreatic adenocarcinomas despite a low incidence of its promoter hypermethylation. ${ }^{37}$ In the current study, a high expression of miR-21 was found to correlate with the downregulation of TIMP3 in pancreatic ductal adenocarcinoma. Therefore, the miR-21-induced downregulation of TIMP3 is likely another oncogenic mechanism in the pancreas.

In summary, our study demonstrated that miR-21 was the most highly expressed miRNA in pancreatic ductal adenocarcinoma, and that the elevated expression of miR-21 was correlated with a worse survival of the patients. The miR-21 expression was closely linked to the immunostaining patterns of tumor suppressors, PDCD4 and TIMP3. Thus, miR21 is suggested to be a key oncogenic miRNA in the pancreas by targeting multiple tumor suppressors and a potential biomarker predicting prognosis of the patients with pancreatic ductal adenocarcinoma.

\section{Acknowledgements}

This work was partly supported by UOEH Grant for Advanced Research (\#H20-1) and Grant-in-Aid for Scientific Research (C) of the Japan Society for the Promotion of Science (\#22590341).

\section{Disclosure/conflict of interest}

The authors declare no conflict of interest.

\section{References}

1 Jemal A, Siegel R, Xu J, et al. Cancer statistics, 2010. CA Cancer J Clin 2010;60:277-300.

2 Tonini G, Pantano F, Vincenzi B, et al. Molecular prognostic factors in patients with pancreatic cancer. Expert Opin Ther Targets 2007;11:1553-1569.

3 Ambros V. MicroRNA pathways in flies and worms: growth, death, fat, stress, and timing. Cell 2003;113: 673-676.

4 Bartel DP. MicroRNAs: genomics, biogenesis, mechanism, and function. Cell 2004;116:281-297.

5 Bloomston M, Frankel WL, Petrocca F, et al. MicroRNA expression patterns to differentiate pancreatic adenocarcinoma from normal pancreas and chronic pancreatitis. JAMA 2007;297:1901-1908.

6 Lee EJ, Gusev Y, Jiang J, et al. Expression profiling identifies microRNA signature in pancreatic cancer. Int J Cancer 2007;120:1046-1054.

7 Feber A, Xi L, Luketich JD, et al. MicroRNA expression profiles of esophageal cancer. J Thorac Cardiovasc Surg 2008;135:255-260.

8 Yanaihara N, Caplen N, Bowman E, et al. Unique microRNA molecular profiles in lung cancer diagnosis and prognosis. Cancer Cell 2006;9:189-198.

9 Schetter AJ, Leung SY, Sohn JJ, et al. MicroRNA expression profiles associated with prognosis and therapeutic outcome in colon adenocarcinoma. JAMA 2008;299:425-436.

10 Qi L, Bart J, Tan LP, et al. Expression of miR-21 and its targets (PTEN, PDCD4, TM1) in flat epithelial atypia of the breast in relation to ductal carcinoma in situ and invasive carcinoma. BMC Cancer 2009;9:163.

11 Selaru FM, Olaru AV, Kan T, et al. MicroRNA-21 is overexpressed in human cholangiocarcinoma and regulates programmed cell death 4 and tissue inhibitor of metalloproteinase 3. Hepatology 2009;49: 1595-1601.

12 Ali S, Almhanna K, Chen W, et al. Differentially expressed miRNAs in the plasma may provide a molecular signature for aggressive pancreatic cancer. Am J Transl Res 2010;3:28-47.

13 Hruban RH, Boffetta P, Hiraoka N, et al. Ductal adenocarcinoma of the pancreas. In: Bosman FT, Carneiro F, Hruban RH, Theise ND (eds). WHO Classification of Tumours of the Digestive System. IARC Press: Lyon, 2010, pp 281-291.

14 Sobin LH, Gospodarowicz MK, Wittekind C, editors. TNM Classification of Malignant Tumours, 7th edn. Wiley-Blackwell: Oxford, 2009, pp 132-135.

15 Hisaoka M, Matsuyama A, Nagao Y, et al. Identification of altered microRNA expression patterns in synovial sarcoma. Genes Chromosomes Cancer 2011;50:137-145.

16 Chan JA, Krichevsky AM, Kosik KS. MicroRNA-21 is an antiapoptotic factor in human glioblastoma cells. Cancer Res 2005;65:6029-6033.

17 Moriyama T, Ohuchida K, Mizumoto K, et al. MicroRNA-21 modulates biological functions of pancreatic cancer cells including their proliferation, invasion, and chemoresistance. Mol Cancer Ther 2009;8: 1067-1074. 
18 Dillhoff M, Liu J, Frankel W, et al. MicroRNA-21 is overexpressed in pancreatic cancer and a potential predictor of survival. J Gastrointest Surg 2008;12: 2171-2176.

19 Zhu S, Wu H, Wu F, et al. MicroRNA-21 targets tumor suppressor genes in invasion and metastasis. Cell Res 2008;18:350-359.

$20 \mathrm{Xu} \mathrm{X}$, Ehdaie B, Ohara N, et al. Synergistic action of Smad4 and Pten in suppressing pancreatic ductal adenocarcinoma formation in mice. Oncogene 2010; 29:674-686.

21 Bhatti I, Lee A, James V, et al. Knockdown of microRNA-21 inhibits proliferation and increases cell death by targeting programmed cell death 4 (PDCD4) in pancreatic ductal adenocarcinoma. J Gastrointest Surg 2011;15:199-208.

22 Chen Y, Knosel T, Kristiansen G, et al. Loss of PDCD4 expression in human lung cancer correlates with tumour progression and prognosis. J Pathol 2003; 200:640-646.

23 Afonja O, Juste D, Das S, et al. Induction of PDCD4 tumor suppressor gene expression by RAR agonists, antiestrogen and HER-2/neu antagonist in breast cancer cells. Evidence for a role in apoptosis. Oncogene 2004;23:8135-8145.

24 Mudduluru G, Medved F, Grobholz R, et al. Loss of programmed cell death 4 expression marks adenomacarcinoma transition, correlates inversely with phosphorylated protein kinase $\mathrm{B}$, and is an independent prognostic factor in resected colorectal cancer. Cancer 2007;110:1697-1707.

25 Hiyoshi Y, Kamohara H, Karashima R, et al. MicroRNA-21 regulates the proliferation and invasion in esophageal squamous cell carcinoma. Clin Cancer Res 2009;15:1915-1922.

26 Motoyama K, Inoue H, Mimori K, et al. Clinicopathological and prognostic significance of PDCD4 and microRNA-21 in human gastric cancer. Int J Oncol 2010;36:1089-1095.
27 Wei NA, Liu SS, Leung TH, et al. Loss of programmed cell death $4(\operatorname{Pdcd} 4)$ associates with the progression of ovarian cancer. Mol Cancer 2009;8:70.

28 Lankat-Buttgereit B, Goke R. The tumour suppressor Pdcd4: recent advances in the elucidation of function and regulation. Biol Cell 2009;101:309-317.

29 Bohm M, Sawicka K, Siebrasse JP, et al. The transformation suppressor protein Pdcd4 shuttles between nucleus and cytoplasm and binds RNA. Oncogene 2003;22:4905-4910.

30 Lankat-Buttgereit B, Goke R. Programmed cell death protein 4 (pdcd4): a novel target for antineoplastic therapy? Biol Cell 2003;95:515-519.

31 Lankat-Buttgereit B, Goke R. Programmed cell death 4 (Pdcd4) - a potential target for new approaches in cancer therapy. Curr Topics Peptide Prot Res 2005;7:63-66.

32 Hayashi A, Aishima S, Miyasaka Y, et al. Pdcd4 expression in intraductal papillary mucinous neoplasm of the pancreas: its association with tumor progression and proliferation. Hum Pathol 2010;41:1507-1515.

33 Blavier L, Henriet P, Imren S, et al. Tissue inhibitors of matrix metalloproteinases in cancer. Ann NY Acad Sci 1999;878:108-119.

34 Ahonen M, Poukkula M, Baker AH, et al. Tissue inhibitor of metalloproteinases-3 induces apoptosis in melanoma cells by stabilization of death receptors. Oncogene 2003;22:2121-2134.

35 Brand K, Baker AH, Perez-Canto A, et al. Treatment of colorectal liver metastases by adenoviral transfer of tissue inhibitor of metalloproteinases-2 into the liver tissue. Cancer Res 2000;60:5723-5730.

36 Ahonen M, Baker AH, Kahari VM. High level expression of tissue inhibitors of metalloproteinases-1,-2 and -3 in melanoma cells achieved by adenovirus mediated gene transfer. Adv Exp Med Biol 1998;451:69-72.

37 Fendrich V, Slater EP, Heinmoller E, et al. Alterations of the tissue inhibitor of metalloproteinase-3 (TIMP3) gene in pancreatic adenocarcinomas. Pancreas 2005; 30:e40-e45. 